International Journal of Quantum Information

(C) World Scientific Publishing Company

\title{
Universal Quantum Gate, Yang-Baxterization and Hamiltonian
}

\author{
Yong Zhang \\ Institute of Theoretical Physics, Chinese Academy of Sciences \\ P. O. Box 2735, Beijing 100080, P. R. China \\ yong@itp.ac.cn \\ Louis H. Kauffman \\ Department of Mathematics, Statistics and Computer Science \\ University of Illinois at Chicago \\ 851 South Morgan Street Chicago, IL, 60607-7045 \\ kauffman@uic.edu \\ Mo-Lin Ge \\ Nankai Institute of Mathematics, Nankai University \\ Tianjin 300071, P. R. China \\ geml@nankai.edu.cn
}

Received 29 December 2004

\begin{abstract}
It is fundamental to view unitary braiding operators describing topological entanglements as universal quantum gates for quantum computation. This paper derives a unitary solution of the quantum Yang-Baxter equation via Yang-Baxterization and constructs the Hamiltonian responsible for the time-evolution of the unitary braiding operator.
\end{abstract}

Keywords: topological entanglement, quantum entanglement, Yang-Baxterization

\section{Introduction}

There are natural relationships between quantum entanglements ${ }^{1}$ and topological entanglements ${ }^{2}$. Topology studies global relationships in spaces, and how one space can be placed within another, such as knotting and linking of curves in three-dimensional space. One way to study topological entanglement and quantum entanglement is to try making direct correspondences between patterns of topological linking and entangled quantum states. The approach of this kind was initiated by Aravind ${ }^{3}$, suggesting that observation of a link would be modelled by deleting one component of the link. But this correspondence property of quantum states and topological links is not basis independent ${ }^{3}$.

A deeper method (we believe) is to consider unitary gates $\check{R}$ that are both universal for quantum computation and are also solutions to the condition for topological braiding. Such $\check{R}$-matrices are unitary solutions to the quantum Yang-Baxter equa- 
tion (QYBE), and can be used as universal quantum gates in the sense discussed in this paper. We are then in a position to compare the topological and quantum properties of these transformations. In this way, we can study the apparently complex relationship among topological entanglement, quantum entanglement, and quantum computational universality. This way has been explored in a series of papers, see Refs. 4-12 for more details.

The present paper is an outgrowth of Ref. 11. It derives the unitary solution of the QYBE via Yang-Baxterization and explores the corresponding dynamical evolution of quantum states. The solutions to the QYBE that we derive by YangBaxterization contain a spectral parameter, and hence do not, except in special cases, give representations of the Artin braid group. These new solutions are unitary, and they do give useful quantum gates. Thus we show in this paper that the full physical subject of solutions to the Yang-Baxter equation (including the spectral parameter) is of interest for quantum computing and quantum information theory.

The plan of the paper is organized as follows. In the second section, we relate a unitary solution of the braid relation to a universal quantum gate. In the third section, we obtain a unitary solution of the QYBE via Yang-Baxterization acting on the solution of the braid relation. In the fourth section, we construct the Hamiltonian responsible for the time-evolution of the braiding operator and write down the Schrödinger equation. In the fifth section, we generate the Bell states by the $\check{R}$-matrix acting on unentangled states and construct the CNOT gate via single qubit transformations acting on the $\check{R}$-matrix. At last, remarks on further research are made.

\section{The QYBE and universal quantum gate}

In this article, the braid group representation (BGR) $b$-matrix ${ }^{5}$ and QYBE solution $\check{R}$-matrix, see Refs. $13-16$, are $n^{2} \times n^{2}$ matrices acting on $V \otimes V$ where $V$ is an $n$-dimensional vector space. As $b$ and $\check{R}$ act on the tensor product $V_{i} \otimes V_{i+1}$, we denote them by $b_{i}$ and $\check{R}_{i}$, respectively.

The BGR $b$-matrix has to satisfy the braid relation

$$
b_{i} b_{i+1} b_{i}=b_{i+1} b_{i} b_{i+1},
$$

while the QYBE has the form

$$
\check{R}_{i}(x) \check{R}_{i+1}(x y) \check{R}_{i}(y)=\check{R}_{i+1} \check{R}_{i}(x y) \check{R}_{i+1}(x)
$$

with the asymptotic condition

$$
\check{R}(x=0)=b,
$$

and $x$ called the spectral parameter. From these two equations both $b$ and $\check{R}(x)$ are fixed up to an overall scalar factor.

A two-qubit gate $G$ is a unitary linear mapping $G: V \otimes V \longrightarrow V$ where $V$ is a two complex dimensional vector space. We say that the gate $G$ is universal for quantum 
computation (or just universal) if $G$ together with local unitary transformations (unitary transformations from $V$ to $V$ ) generates all unitary transformations of the complex vector space of dimension $2^{n}$ to itself. It is well-known ${ }^{1}$ that the CNOT gate is a universal gate.

A gate $G$, as above, is said to be entangling if there is a vector

$$
|\alpha \beta\rangle=|\alpha\rangle \otimes|\beta\rangle \in V \otimes V
$$

such that $G|\alpha \beta\rangle$ is not decomposable as a tensor product of two qubits. Under these circumstances, one says that $G|\alpha \beta\rangle$ is entangled.

In Refs. 17-18, the Brylinskis give a general criterion of $G$ to be universal. They prove that a two-qubit gate $G$ is universal if and only if it is entangling.

In Ref. 11, Kauffman and Lomonaco prove the following result.

Theorem 1. Let

$$
\check{R}=\left(\begin{array}{cccc}
1 / \sqrt{2} & 0 & 0 & 1 / \sqrt{2} \\
0 & 1 / \sqrt{2} & -1 / \sqrt{2} & 0 \\
0 & 1 / \sqrt{2} & 1 / \sqrt{2} & 0 \\
-1 / \sqrt{2} & 0 & 0 & 1 / \sqrt{2}
\end{array}\right)
$$

be the unitary solution to the braid relation (1). Then $\check{R}$ is a universal gate. The proof below (repeated from Ref. 11) gives a specific expression for the CNOT gate in terms of $\check{R}$.

Proof. This result follows at once from the Brylinksis' Theorem ${ }^{18}$, since $\check{R}$ is highly entangling. For a direct computational proof, it suffices to show that the CNOT gate can be generated from $\check{R}$ and local unitary transformations. Let

$$
\begin{aligned}
\alpha=\left(\begin{array}{ll}
1 / \sqrt{2} & 1 / \sqrt{2} \\
1 / \sqrt{2} & -1 / \sqrt{2}
\end{array}\right), & \beta=\left(\begin{array}{cc}
-1 / \sqrt{2} & 1 / \sqrt{2} \\
i / \sqrt{2} & i / \sqrt{2}
\end{array}\right) \\
\gamma=\left(\begin{array}{ll}
1 / \sqrt{2} & i / \sqrt{2} \\
1 / \sqrt{2} & -i / \sqrt{2}
\end{array}\right), & \delta=\left(\begin{array}{ll}
1 & 0 \\
0 & i
\end{array}\right) .
\end{aligned}
$$

Let $M=\alpha \otimes \beta$ and $N=-\gamma \otimes \delta$. Then it is straightforward to verify that

$$
\mathrm{CNOT}=M \cdot \check{R} \cdot N
$$

This completes the proof. 


\section{The unitary $\check{R}(x)$-matrix via Yang-Baxterization}

The QYBE (2) solution $\check{R}$-matrices usually depend on the deformation parameter $q$ and the spectral parameter $x$. With two such parameters, there exist two approaches to solving the QYBE (2). Taking the limit of $x \rightarrow 0$ leads to the braid relation (1) from the QYBE (2) and the BGR $b$-matrix from the $\check{R}$-matrix. Concerning relations between the BGR and $x$-dependent solutions of the QYBE (2), we either reduce a known $\check{R}(x)$-matrix to a BGR $b$-matrix, see Refs. $20-22$, or construct a $\check{R}(x)$-matrix from a given BGR $b$-matrix. Such a construction is called Yang-Baxterization.

In knot theory, these solutions were first studied by Jones ${ }^{19}$ and Turaev ${ }^{22}$ for the BGR satisfying the Heck algebra relations and for the BGR satisfying the Birman-Wenzl algebra relations (corresponding to the Kauffman two-variable polynomial ${ }^{2}$ ). Later the more general cases with a BGR having three or four unequal eigenvalues were considered in Refs. 23-25, including all known trigonometric solutions to the QYBE. Also Yang-Baxterization of non-standard BGR $b$-matrix has been discussed in Refs. 26-33.

In this section, we apply Yang-Baxterization to derive a unitary $\check{R}(x)$-matrix so that it is possible to find out the Hamiltonian controlling the evolution of quantum entangled states. As an example, we will present a solution of the BGR for the eightvertex model and its corresponding unitary $\check{R}$-matrix via Yang-Baxterization.

In terms of non-vanishing Boltzman weights $w_{1}, \cdots, w_{8}$, the BGR $b$-matrix of the eight-vertex model assumes the form

$$
b=\left(\begin{array}{cccc}
w_{1} & 0 & 0 & w_{7} \\
0 & w_{5} & w_{3} & 0 \\
0 & w_{4} & w_{6} & 0 \\
w_{8} & 0 & 0 & w_{2}
\end{array}\right) .
$$

Choosing suitable Boltzman weights leads to solutions of the braid relation (1).

Setting $w_{1}=w_{2}=w_{5}=w_{6}$ gives us $w_{1}^{2}=w_{3}^{2}=w_{4}^{2}$ and $w_{3}^{2}+w_{7} w_{8}=0$. In the case of $w_{3} \neq w_{4}$, we have $w_{3}=-w_{4}$ and $w_{1}= \pm w_{3}$. The BGR $b$-matrix has the form

$$
b_{ \pm}=\left(\begin{array}{cccc}
w_{1} & 0 & 0 & w_{7} \\
0 & w_{1} & \pm w_{1} & 0 \\
0 & \mp w_{1} & w_{1} & 0 \\
-\frac{w_{1}^{2}}{w_{7}} & 0 & 0 & w_{1}
\end{array}\right) \Longleftrightarrow\left(\begin{array}{cccc}
1 & 0 & 0 & q \\
0 & 1 & \pm 1 & 0 \\
0 & \mp 1 & 1 & 0 \\
-q^{-1} & 0 & 0 & 1
\end{array}\right)
$$

It has two eigenvalues $\lambda_{1}=1-i$ and $\lambda_{2}=1+i$. The corresponding $\check{R}(x)$-matrix via Yang-Baxterization is obtained to be

$$
\begin{aligned}
\check{R}_{ \pm}(x) & =b_{ \pm}+x \lambda_{1} \lambda_{2} b_{ \pm}^{-1} \\
& =\left(\begin{array}{cccc}
1+x & 0 & 0 & q(1-x) \\
0 & 1+x & \pm(1-x) & 0 \\
0 & \mp(1-x) & 1+x & 0 \\
-q^{-1}(1-x) & 0 & 0 & 1+x
\end{array}\right) .
\end{aligned}
$$


Assume the spectral parameter $x$ and the deformation parameter $q$ to be complex numbers. The unitarity condition

$$
\check{R}_{ \pm}(x) \check{R}_{ \pm}^{\dagger}(x)=\check{R}_{ \pm}^{\dagger}(x) \check{R}_{ \pm}(x) \propto \rho_{ \pm} \mathbb{1}
$$

leads to the following equations

$$
\left\{\begin{array}{cl}
\|1+x\|^{2}+\|q\|^{2}\|1-x\|^{2} & =\rho_{ \pm} \\
\|1+x\|^{2}+\frac{1}{\|q\|^{2}}\|1-x\|^{2} & =\rho_{ \pm} \\
\|1+x\|^{2}+\|1-x\|^{2} & =\rho_{ \pm} \\
(1-x)(1+\bar{x})-(1+x)(1-\bar{x}) & =0 \\
-q^{-1}(1-x)(1+\bar{x})+\bar{q}(1+x)(1-\bar{x}) & =0
\end{array}\right.
$$

which specify $x$ real and $q$ living at a unit circle.

Introducing the new variables of angles $\theta$ and $\varphi$ as follows

$$
\cos \theta=\frac{1}{\sqrt{1+x^{2}}}, \quad \sin \theta=\frac{x}{\sqrt{1+x^{2}}}, \quad q=e^{-i \varphi},
$$

we represent the $\check{R}_{ \pm}(x)$-matrix in a new form

$$
\check{R}_{ \pm}(\theta)=\cos \theta b_{ \pm}(\varphi)+\sin \theta\left(b_{ \pm}\right)^{-1}(\varphi)
$$

in which the BGR $b_{ \pm}(\varphi)$-matrix is given by

$$
b_{ \pm}(\varphi)=\frac{1}{\sqrt{2}}\left(\begin{array}{cccc}
1 & 0 & 0 & e^{-i \varphi} \\
0 & 1 & \pm 1 & 0 \\
0 & \mp 1 & 1 & 0 \\
-e^{i \varphi} & 0 & 0 & 1
\end{array}\right)
$$

Taking $\theta=\varphi=0$, the universal quantum gate $\check{R}$-matrix (4) is derived.

\section{The constructions of the Hamiltonian}

In this section, we present a method of constructing the Hamiltonian from the above unitary $\check{R}_{ \pm}(x)$-matrix (8) or $\check{R}_{ \pm}(\theta)$-matrix (12) for the eight-vertex model.

In terms of the unitary QYBE solution $\check{R}(x)$ (8), we construct the timeindependent Hamiltonian $H_{ \pm}$having the form

$$
H_{ \pm}=\left.i \frac{\partial}{\partial x}\left(\rho_{ \pm}^{-\frac{1}{2}} \breve{R}_{ \pm}\right)\right|_{x=1}=-\frac{i}{2} b_{ \pm}^{2}=\frac{i}{2}\left(\begin{array}{cccc}
0 & 0 & 0 & -e^{-i \varphi} \\
0 & 0 & \mp 1 & 0 \\
0 & \pm 1 & 0 & 0 \\
e^{i \varphi} & 0 & 0 & 0
\end{array}\right)
$$

Interestingly, we have the "time-dependent" Hamiltonian $H_{ \pm}(x)$ by

$$
H_{ \pm}(x)=i \frac{\partial}{\partial x}\left(\rho_{ \pm}^{-\frac{1}{2}} \check{R}_{ \pm}\right) \rho_{ \pm}^{-\frac{1}{2}} \check{R}_{ \pm}^{\dagger}(x)=-\frac{i}{1+x^{2}} b_{ \pm}^{2}
$$

which derives the above Hamiltonian $H_{ \pm}$at $x=1$. When $x$ is real, the Hamiltonian $H_{ \pm}(x)$ is a Hermition operator. 
6 Yong Zhang, Louis H. Kauffman, Mo-Lin Ge

The wave function $\psi(x)$ is specified by the $\check{R}(x)$-matrix, with $\psi(x)=\check{R}(x) \psi$, the pure state $\psi$ independent of the time (or the spectral parameter $x$ ). Hence we obtain the Shrödinger equation corresponding to the time evolution of $\psi(x)$ controlled by the $\check{R}(x)$-matrix,

$$
i \frac{\partial \psi(x)}{\partial x}=H(x) \psi(x)
$$

For simplicity, we study the unitary $\check{R}(\theta)$-matrix (12) to decide the unitary evolution of quantum states. After some algebra, the Hamiltonian $H_{ \pm}$is obtained to be

$$
H_{ \pm}=\frac{i}{2} \frac{\partial}{\partial \theta}\left(\rho_{ \pm}^{-\frac{1}{2}} \check{R}_{ \pm}\right) \rho_{ \pm}^{-\frac{1}{2}} \check{R}_{ \pm}^{\dagger}=\frac{i}{2} \frac{\partial x}{\partial \theta} H_{ \pm}(x)=-\frac{i}{2} b_{ \pm}^{2}
$$

which is independent of the time variable $\theta$. So we have the expected Schrödinger equation

$$
i \frac{\partial \psi_{ \pm}(\theta)}{\partial \theta}=H_{ \pm} \psi_{ \pm}(\theta)
$$

In terms of the Pauli matrices $\sigma_{x}, \sigma_{y}$ and $\sigma_{z}$ and $\sigma_{ \pm}=\frac{1}{2}\left(\sigma_{x} \pm i \sigma_{y}\right)$, the Hamiltonian (17) has the form ${ }^{\text {a }}$

$$
H_{ \pm}=\frac{i}{2}\left(-e^{-i \varphi} \sigma_{+} \otimes \sigma_{+}+e^{i \varphi} \sigma_{-} \otimes \sigma_{-} \mp \sigma_{+} \otimes \sigma_{-} \pm \sigma_{-} \otimes \sigma_{+}\right) .
$$

Introducing the two-dimensional vector $\vec{\sigma}$ and two unit directional vector $\vec{n}_{1}$ and $\vec{n}_{2}$ in $x y$-plane:

$$
\vec{\sigma}=\left(\sigma_{x}, \sigma_{y}\right) ; \quad \vec{n}_{1}=\left(\cos \frac{\pi+\varphi}{2}, \sin \frac{\pi+\varphi}{2}\right), \vec{n}_{2}=\left(\cos \frac{\varphi}{2}, \sin \frac{\varphi}{2}\right),
$$

the projections of the vector $\vec{\sigma}$ into $\vec{n}_{1}$ and $\vec{n}_{2}$ are given by

$$
\begin{aligned}
\sigma_{n_{1}} & =\vec{\sigma} \cdot \vec{n}_{1}=\sigma_{+} e^{-\frac{i}{2}(\varphi+\pi)}+\sigma_{-} e^{\frac{i}{2}(\varphi+\pi)}, \\
\sigma_{n_{2}} & =\vec{\sigma} \cdot \vec{n}_{2}=\sigma_{+} e^{-\frac{i}{2} \varphi}+\sigma_{-} e^{\frac{i}{2} \varphi} .
\end{aligned}
$$

The Hamiltonian (19) can be recast to

$$
H_{+}=\frac{1}{2} \sigma_{n_{1}} \otimes \sigma_{n_{2}}, \quad H_{-}=\frac{1}{2} \sigma_{n_{2}} \otimes \sigma_{n_{1}} .
$$

We consider the unitary time-evolutional operator $U_{ \pm}(\theta)$ determined by the Hamiltonian $H_{ \pm}$, for example, $U_{+}$given by

$$
U_{+}(\theta)=e^{-\frac{i}{2}\left(\sigma_{n_{1}} \otimes \sigma_{n_{2}}\right) \theta}=\cos \frac{\theta}{2}-i \sin \frac{\theta}{2} \sigma_{n_{1}} \otimes \sigma_{n_{2}} .
$$




\section{The Bell states and CNOT gate}

Now we discuss physics related to the time-evolution of the universal quantum gate determined by the unitary $\check{R}(\theta)$-matrix (12). The braid group representation $b_{ \pm}(\varphi)$-matrix (13) yields the Bell states with the phase factor $e^{-i \varphi}$,

$$
b_{ \pm}(\varphi)\left(\begin{array}{c}
|00\rangle \\
|01\rangle \\
|10\rangle \\
|11\rangle
\end{array}\right)=\frac{1}{\sqrt{2}}\left(\begin{array}{c}
|00\rangle-e^{i \varphi}|11\rangle \\
|01\rangle \mp|10\rangle \\
\pm|01\rangle+|10\rangle \\
e^{-i \varphi}|00\rangle+|11\rangle
\end{array}\right)
$$

which shows that $\varphi=0$ leads to the Bell states, the maximum of entangled states,

$$
\frac{1}{\sqrt{2}}(|00\rangle \pm|11\rangle), \quad \frac{1}{\sqrt{2}}(|10\rangle \pm|01\rangle) .
$$

In terms of the Hamiltonian $H_{ \pm}(17)$, the $\check{R}_{ \pm}(\theta)$-matrix (12) has the form

$$
\check{R}_{ \pm}(\theta)=\cos \left(\frac{\pi}{4}-\theta\right)+2 i \sin \left(\frac{\pi}{4}-\theta\right) H_{ \pm}=e^{i\left(\frac{\pi}{2}-2 \theta\right) H_{ \pm}}
$$

which can be also used to construct the CNOT gate with additional single qubit transformations, examples see Ref. 11 . The $\check{R}$-matrix (4) is realized as

$$
\check{R}=\left.\check{R}_{-}(\theta)\right|_{\theta=\varphi=0}=e^{i \frac{\pi}{4}\left(\sigma_{x} \otimes \sigma_{y}\right)} .
$$

Applying the unitary $\check{R}(\theta)$-matrix (12) to unentangled states, we have

$$
\check{R}_{ \pm}(\theta)\left(\begin{array}{l}
|00\rangle \\
|01\rangle \\
|10\rangle \\
|11\rangle
\end{array}\right)=\left(\begin{array}{l}
\cos \left(\frac{\pi}{4}-\theta\right)|00\rangle-e^{i \varphi} \sin \left(\frac{\pi}{4}-\theta\right)|11\rangle \\
\cos \left(\frac{\pi}{4}-\theta\right)|01\rangle \mp \sin \left(\frac{\pi}{4}-\theta\right)|10\rangle \\
\left.\cos \left(\frac{\pi}{4}-\theta\right)|10\rangle \pm \sin \left(\frac{\pi}{4}-\theta\right)|01\rangle\right) \\
\cos \left(\frac{\pi}{4}-\theta\right)|11\rangle+e^{-i \varphi} \sin \left(\frac{\pi}{4}-\theta\right)|00\rangle
\end{array}\right) .
$$

Hence with the Bloch vectors on the Bloch sphere ${ }^{1}$, the variables $\theta$ and $\varphi$ realize their geometric meanings so that the construction of the CNOT gate becomes clear.

To obtain other two-qubit quantum gates, for instance the CNOT gate, we have to apply single qubit unitary transformations $A, B, C, D$ which can be possibly found in the Bloch sphere ${ }^{1}$ by $S O(3)$ rotations, namely,

$$
(A \otimes B) U_{ \pm}(\theta)(C \otimes D)=P_{\uparrow} \otimes \mathbb{1}+P_{\downarrow} \otimes \sigma_{x}=\mathrm{CNOT}
$$

where the states $|\uparrow\rangle$ and $|\downarrow\rangle$ are the eigenvectors of $\sigma_{z}, \sigma_{z}|\uparrow\rangle=|\uparrow\rangle, \sigma_{z}|\downarrow\rangle=-|\downarrow\rangle$ and the projection operators $P_{\uparrow}$ and $P_{\downarrow}$ have the forms

$$
P_{\uparrow}=|\uparrow\rangle\left\langle\uparrow\left|, \quad P_{\downarrow}=\right| \downarrow\right\rangle\langle\downarrow| .
$$

Define the $S O(3)$ rotation around the $\vec{n}$-axis by

$$
D_{\vec{n}}(\theta)=e^{-\frac{i}{2}(\vec{\sigma} \cdot \vec{n}) \theta}
$$

where $\vec{\sigma}=\left(\sigma_{x}, \sigma_{y}, \sigma_{z}\right)$. For examples:

$$
D_{z}\left(-\frac{\varphi}{2}\right)=e^{i \frac{\varphi}{4} \sigma_{z}}, D_{x}\left(\frac{\pi}{2}\right)=e^{-i \frac{\pi}{4} \sigma_{x}}, D_{y}\left(\frac{\pi}{2}\right)=e^{-i \frac{\pi}{4} \sigma_{y}}
$$


satisfy

$$
D_{x}\left(\frac{\pi}{2}\right) D_{z}\left(-\frac{\varphi}{2}\right) \sigma_{n_{1}} D_{z}\left(\frac{\varphi}{2}\right) D_{x}\left(-\frac{\pi}{2}\right)=\sigma_{z}, \quad D_{z}\left(-\frac{\varphi}{2}\right) \sigma_{n_{2}} D_{z}\left(\frac{\varphi}{2}\right)=\sigma_{x} .
$$

Consider the evolution operator $U_{+}(\theta)$. Choosing suitable single qubit transformations, we obtain

$$
\left(D_{x}\left(\frac{\pi}{2}\right) D_{z}\left(-\frac{\varphi}{2}\right) \otimes D_{z}\left(-\frac{\varphi}{2}\right)\right) U_{+}(\theta)\left(D_{z}\left(\frac{\varphi}{2}\right) D_{x}\left(-\frac{\pi}{2}\right) \otimes D_{z}\left(\frac{\varphi}{2}\right)\right)=e^{-\frac{i}{2}\left(\sigma_{z} \otimes \sigma_{x}\right) \theta}
$$

which has another form

$$
e^{-\frac{i}{2}\left(\sigma_{z} \otimes \sigma_{x}\right) \theta}=P_{\uparrow} \otimes e^{-\frac{i}{2} \sigma_{x} \theta}+P_{\downarrow} \otimes e^{\frac{i}{2} \sigma_{x} \theta} .
$$

Set $\theta=\frac{\pi}{2}$. To construct the CNOT gate, we need additional single qubit transformations

$$
\left(\delta \otimes e^{i \frac{\pi}{4} \sigma_{x}}\right) e^{-i \frac{\pi}{4}\left(\sigma_{z} \otimes \sigma_{x}\right)}=\mathrm{CNOT}
$$

in which the phase gate $\delta$ has the form $\delta=P_{\uparrow}-i P_{\downarrow}$, see (5).

Consider the evolution operator $U_{-}\left(\theta=-\frac{\pi}{2}, \varphi=0\right)$, namely the $\check{R}$-matrix (4) given by $e^{i \frac{\pi}{4}\left(\sigma_{x} \otimes \sigma_{y}\right)}$ which is transformed into $e^{i \frac{\pi}{4}\left(\sigma_{z} \otimes \sigma_{x}\right)}$ by

$$
\left(D_{y}\left(-\frac{\pi}{2}\right) \otimes D_{z}\left(-\frac{\pi}{2}\right)\right) e^{i \frac{\pi}{4}\left(\sigma_{x} \otimes \sigma_{y}\right)}\left(D_{y}\left(\frac{\pi}{2}\right) \otimes D_{z}\left(\frac{\pi}{2}\right)\right)=e^{i \frac{\pi}{4}\left(\sigma_{z} \otimes \sigma_{x}\right)} .
$$

So we obtain another proof for Theorem 1 in the second section or Ref. 11.

\section{Concluding remarks}

Motivated by the observation that there are certain natural similarities between quantum entanglements and topological entanglements, we derive the unitary solution of the QYBE or the unitary $\check{R}(x)$-matrix via Yang-Baxterization and construct the related Hamiltonian for the eight-vertex model. With the Brylinksis' Theorem ${ }^{18}$, the unitary $\check{R}(x)$-matrix (8) is also a universal quantum gate except for $x=1$.

In the forth-coming article ${ }^{34}$, we obtain more unitary $\check{R}(x)$ solutions determining the evolution of universal quantum gates, and we study Yang-Baxterizations of the standard or non-standard representations of the six-vertex model and the complete solutions of the non-vanishing eight-vertex models.

\section{Acknowledgements}

Y. Zhang thanks speakers for helpful lectures in the Fourth International Wihelm and Else Heraeus Summer School on "Fundamentals of Quantum Information Processing" in Wittenberg, and thanks P. Zhang for patiently answering him questions on universal quantum gates. We thank C.M. Bai and J.M. Hou for helpful discussions. This work is in part supported by NSFC-10447134.

For L.H. Kauffman, most of this effort was sponsored by the Defense Advanced Research Projects Agency (DARPA) and Air Force Research Laboratory, Air Force Materiel Command, USAF, under agreement F30602-01-2-05022. The U.S. Government is authorized to reproduce and distribute reprints for Government purposes 
notwithstanding any copyright annotations thereon. The views and conclusions contained herein are those of the authors and should not be interpreted as necessarily representing the official policies or endorsements, either expressed or implied, of the Defense Advanced Research Projects Agency, the Air Force Research Laboratory, or the U.S. Government. (Copyright 2004.) It gives L.H. Kauffman great pleasure to acknowledge support from NSF Grant DMS-0245588.

\section{References}

1. M. Nielsen and I. Chuang, Quantum Computation and Quantum Information (Cambridge University Press, 1999).

2. L. H. Kauffman, Knots and Physics (World Scientific Publishers, 2002).

3. P. K. Aravind, Borromean entanglement of the GHZ state, in Potentiality, Entanglement and Passion-at-a-Distance, eds. Cohen et al. (Kluwer Academic Publishers, Boston 1997).

4. L. H. Kauffman, in Quantum Computation and Information, eds. S. Lomonaco Jr. (AMS CONM/305, 2002), pp. 101-137.

5. L. H. Kauffman and S. J. Lomonaco Jr., New Journal of Physics 4 (2002) 73.1-73.18.

6. L. H. Kauffman and S. J. Lomonaco Jr., in Quantum Information and ComputationSpie Proceedings, 21-22 April, 2003, Orlando, FL, eds. Donkor, Pinch and Brandt, Vol. 5105, pp. 51-58.

7. L. H. Kauffman, in Quantum Computation, eds. S. Lomonaco (AMS PSAPM/58, 2002), pp. 273-303.

8. L. H. Kauffman and S. J. Lomonaco Jr., Comparing quantum entanglement and topological entanglement, quant-ph/0205137.

9. L. H. Kauffman and S. J. Lomonaco Jr., Quantum knots, quant-ph/0403228.

10. L. H. Kauffman, Teleportation topology, quant-ph/0407224.

11. L. H. Kauffman and S. J. Lomonaco Jr., New Journal of Physics 6 (2004) 134.

12. H. Dye, Quantum Information Processing 2 (2003) 117-150.

13. C. N. Yang, Phys. Rev. Lett. 19 (1967) 1312-1314.

14. R. J. Baxter, Annals Phys. 70 (1972) 193-228.

15. L. D. Faddeev, Integrable models in (1+1)-dimensional quantum field theory, in Proc. of Summer School of Theoretical Physics, Les Houches, 1982 (Elsevier, Amsterdam, 1984).

16. M. Jimbo, Adv. Ser. Math. Phys. 10 (1989) 1.

17. M. J. Bremmer et al., Phys. Rev. Lett. 89 (2002) 247902.

18. J. L. Brylinski and R. Brylinski, universal quantum gates, in Mathematics of Quantum Computation, eds. R. Brylinski and G. Chen (Chapman \& Hall/CRC Press, Boca Raton, Florida, 2002).

19. V.F.R. Jones, Int. J. Mod. Phys. A6 (1991) 2035-2043.

20. Y. Akutsu and M. Wadati, J. Phys. Soc. Jap. 56 (1987) 3039-3051.

21. Y. Akutsu, T. Deguchi and M. Wadati, J. Phys. Soc. Jap. 56 (1987) 3464-3479.

22. V. G. Turaev, Invent. Math. 92 (1988) 527.

23. M. L. Ge, K. Xue and Y. S. Wu, in Braid Goup, Knot Theory and Statistical Mechanics, eds. C. N. Yang and M. L. Ge (World Scientific, Singapore, 1994), pp. 130-152.

24. Y. Cheng, M.L. Ge and K. Xue, Commun. Math. Phys. 136 (1991) 195.

25. M.L. Ge, Y.S. Wu and K. Xue, Int. J. Mod. Phys 6 A (1991) 3735.

26. K. Sogo, M. Uchinami, Y. Akutsu and M. Wadati, Prog. Theor. Phys. 68 (1982).

27. M. L. Ge, L. H. Gwa and H. K. Zhao, J. Phys. A: Math. Gen. 23 (1990) L 795-L 798.

28. M. Couture, Y. Cheng, M. L. Ge and K. Xue, Int. J. Mod. Phys. A6 (1991) 559. 
10 Yong Zhang, Louis H. Kauffman, Mo-Lin Ge

29. N. H. Jing, M. L. Ge and Y.S. Wu, Lett. Math. Phys. 21 (1991) 183.

30. M. L. Ge and K. Xue, J. Math. Phys. 32 (1991) 1301-1309.

31. H. C. Lee, Physics, geometry, and topology, in Proc. of the NATO Advanced Study Institute and Banff Summer School in Theoretical Physics (Plemum. N.Y. 1991).

32. H. C. Lee and M. Couture, A method to construct closed braids from links and a new polynomial for connected Links, Chalk River Preprint, Canada (1988).

33. C. L. Schultz, Phys. Rev. Lett. 46 (1981) 629.

34. Y. Zhang, L. H. Kauffman and M. L. Ge, Yang-Baxterizations, universal quantum gates and Hamiltonians, quant-ph/0502015. 